\title{
RESPUESTA A LA ADICIÓN DE NITRÓGENO Y FÓSFORO EN EL CRECIMIENTO DE TYPHA DOMINGENSIS Y SCHOENOPLECTUS AMERICANUS
}

\author{
Yazmín Escutia-Lara ${ }^{1}$, Erick de la Barrera², Yanira Martínez-de la Cruz \\ Y ROBERTO LINDIG-CISNEROS 3,4 \\ ${ }^{1}$ Facultad de Biología, Universidad Michoacana de San Nicolás de Hidalgo \\ ${ }^{2}$ Centro de Investigaciones en Ecosistemas, Universidad Nacional Autónoma de México \\ ${ }^{3}$ Laboratorio de Ecología de Restauración, Centro de Investigaciones en Ecosistemas, \\ Universidad Nacional Autónoma de México \\ ${ }^{4}$ Autor para la correspondencia: rlindig@oikos.unam.mx
}

\begin{abstract}
Resumen: La respuesta a los cambios en las concentraciones de nutrimentos en las plantas de los humedales puede determinar la dinámica entre ellas. Estudios previos con Typha domingensis (Typhaceae) muestran discrepancias en cuanto a su respuesta al nitrógeno y al fósforo; en comparación, de Schoenoplectus americanus (Cyperaceae), una especie con la que coexiste con la primera en humedales del occidente de México, no existe información. En este trabajo se llevaron a cabo dos experimentos para cuantificar la respuesta de estas dos especies a diferentes concentraciones de N y P así como la combinación de ambos en el crecimiento y en particular sobre la acumulación de almidón en los rizomas. Los resultados indican que $T$. domingensis responde principalmente a la adición de $\mathrm{N}$ con un mayor crecimiento, número de hojas y biomasa mientras que $S$. americanus responde a la adición de $\mathrm{N}$ con un mayor crecimiento y número de hojas aunque para la acumulación de biomasa aérea responde a la adición de ambos nutrientes. Para S. americanus, a diferencia de T. domingensis, ningún tratamiento de fertilización tuvo un efecto diferencial en la acumulación de almidón. Los resultados de este estudio sugieren que si se incrementan las concentraciones de nitrógeno en humedales en donde coexisten Typha domingensis y Schoenoplectus americanus la primera podría desplazar a la segunda, causando pérdida de la biodiversidad de los mismos
\end{abstract}

Palabras clave: humedal, nutrimentos, crecimiento, hidrófitas

\begin{abstract}
Responses to nutrient concentrations can determine the dynamics among wetland plant species. One of these species is Typha domingensis (Typhaceae) for which previous studies have obtained dissimilar results on the response to additions of nitrogen and phosphorus. Schoenoplectus americanus (Cyperaceae), is a species that coexists with the latter in Western Mexico, and no data has been gathered for responses to these nutrients. In this study two experiments were carried out to quantify response of the two species to addition of $\mathrm{N}$ and $\mathrm{P}$ as well as both nutrients combined. Results showed that $T$. domingensis responds mostly to $\mathrm{N}$ addition increasing height and particularly by accumulating starch in the rhizomes, and that $S$. americanus responds to $\mathrm{N}$ addition by increasing height and number of leaves, but aerial biomass accumulation responds to both nutrients. For $S$. americanus, in contrast with $T$. domingensis, starch accumulation did not respond to nutrient treatments. Our results suggest that if nitrogen concentrations are increased in wetlands where Typha domingensis and Schoenoplectus americanus coexist, the first species might replace the second cuasing biodiversity loss.
\end{abstract}

Key words: wetland, nutrients, hydrophytes

$\mathbf{N}$ umerosas especies que crecen en los humedales responden a los aportes de nutrientes, particularmente fósforo y nitrógeno, alterando sus tasas de crecimiento de forma considerable (Macek y Rejmánková, 2007; Woo y Zedler, 2002). La respuesta de las plantas a los nutrientes es bien conocida, en el caso de las plantas de los humedales, las respuestas varían dependiendo de las características edáficas del humedal. En términos generales, las plantas de los humedales costeros son más susceptibles a las variaciones en nitrógeno y las plantas de humedales de aguas continentales a las variaciones en fósforo (Cronk y Fennessy, 2001). Sin embargo, debido a las características fisicoquímicas de 
los suelos hídricos varios procesos, como la desnitrificación, pueden alterar la dinámica de los nutrimentos esenciales para las plantas (Richardson y Vepraskas, 2001).

En México se encuentran diversos tipos de humedales que cubren $0.66 \%$ del territorio nacional, y en su mayoría se concentran en las regiones costeras, en particular en la costa del Golfo de México (Montes-Cartes et al., 1999; Flores Verdugo et al, 2007; Lara-Lara et al., 2008; Peralta-Pelaez y MorenoCasasola 2009). Sin embargo, también se encuentran humedales de importancia en la región continental del occidente del país, en particular los asociados a los mayores cuerpos de agua como son Chapala, Cuitzeo, Pátzcuaro y Yuriria. En particular en los lagos de Cuitzeo y Yuriria, la estructura de la vegetación acuática ha sido descrita (Ramos-Ventura y Novelo-Retana, 1993; Rojas-Moreno y Novelo-Retana, 1995), y en estos humedales destacan como especies dominantes especies de los géneros Typha, Scirpus y Schoenoplectus.

Entre las especies que responden de manera notable a los cambios en la disponibilidad de nutrimentos encontramos a aquellas que pertenecen al género Typha (Typhaceae) que incrementan su cobertura cuando los aportes de nitrógeno y/o fósforo se incrementan (Shih y Finkelstein, 2008). En particular, Typha domingensis Presl., es una especie característica de muchos humedales de agua dulce en el occidente de México que en muchos sitios forma un patrón de zonación característico con Schoenoplectus americanus (Pers.) Volkart ex Schinz and Keller (Cyperaceae) (Ramos Ventura y Novelo Retana, 1993; Rojas Moreno y Novelo Retana, 1995). Si los aportes de nutrientes se incrementan es posible que $T$. domingensis desplace a $S$. americanus como ha ocurrido en otras regiones (Craft et al., 1995), debido a que esta especie, como otras del mismo género, pudiera presentar un comportamiento invasor en respuesta a alteraciones en el régimen de nutrientes (Woo y Zedler, 2002; Galatowitsch et al., 1999; Zedler y Kercher, 2004). En los humedales en donde se encuentra una zonación entre $T$. domingensis y $S$. americanus, la diversidad vegetal depende en buena medida de la dinámica de la distribución de las especies dominantes (Escutia-Lara et al., 2009), por lo que entender la respuesta de estas dos especies a las concentraciones de nutrimentos en el medio es de gran importancia para comprender y en su caso manejar la dinámica de los humedales.

En condiciones experimentales Typha domingensis creciendo en una matriz de $S$. americanus responde a la adición de nitrógeno pero no a la adición de fósforo, tanto en el número de rizomas formados durante una temporada de crecimiento como en la biomasa de los mismos, aunque no existen diferencias en la biomasa aérea (Escutia-Lara et al., 2009). Estos resultados contrastan con otros que han mostrado que $T$. domingensis responde a la adición de fósforo (Macek y Rejmmankova, 2007; Lorenzen et al., 2001; Miao et al., 2000). Un análisis en los rizomas de S. americanus indicó presencia de almidón y en el género Schoenoplectus sólo se reportó presencia de almidón en S. lacustris (Stein- mann y Brandle, 1984).

El presente estudio se condujo con el propósito de determinar el efecto de la adición de nitrógeno y fósforo en Typha domingensis y Schoenoplectus americanus, establecidas experimentalmente en forma independientemente, en crecimiento, acumulación de biomasa y de almidón en los rizomas, para conocer la respuesta de estas dos especies a los nutrientes antes mencionados y de esta manera poder entender mejor la dinámica entre ellas.

\section{Materiales y Métodos}

Para el presente estudio se extrajeron 216 rizomas de Schoenoplectus americanus y 216 de Typha domingensis del manantial de La Mintzita ubicado al suroeste de la Ciudad de Morelia, Michoacán México, $19^{\circ}$ 8'43" N, y 101 7'47” W. Los rizomas se seleccionaron para que su peso fresco no variara más de $5 \%$ entre sí. Se utilizaron 24 mesocosmos (tinas de plástico) de 2101 de capacidad, 12 para cada especie. Dentro de cada uno se colocaron 9 macetas de 51 de capacidad ( $25 \mathrm{~cm}$ de altura $\times 16 \mathrm{~cm}$ de diámetro), elevadas $5 \mathrm{~cm}$ sobre el fondo del mesocosmo para facilitar el movimiento del agua. En cada maceta se transplantó un rizoma. Los mesocosmos fueron nivelados y una válvula de flotador mantuvo el nivel de agua constante en cada uno.

Los tratamientos de fertilización consistieron en un control y la aplicación de $\mathrm{KH}_{2} \mathrm{PO}_{4}$ en dos cantidades, 0.068 y $0.137 \mathrm{~g}$, y de $\mathrm{KNO}_{3}$ en dos cantidades, 0.15 y $0.30 \mathrm{~g}$, el diseño experimental fue ortogonal para cada especie de tal forma que se contó con 9 tratamientos y 12 repeticiones por tratamiento. El fertilizante se aplicó en la superficie de cada maceta disuelto en $15 \mathrm{ml}$ de agua destilada. Para el tratamiento control se agregaron $15 \mathrm{ml}$ de agua destilada. La adición de nutrimentos fue cada 15 días durante 6 meses (mayo a octubre). Se eligieron las sales de potasio porque es sabido que este elemento no causa efectos tóxicos en las plantas aun en altas concentraciones (Núñez Escobar 2007). Las variables a medir mensualmente fueron altura de tallo y su número. Al final del experimento se cortaron todos los tallos y se extrajeron los rizomas. La biomasa se colocó en una estufa a $60{ }^{\circ} \mathrm{C}$ para uniformar con peso seco. El análisis de la concentración de almidón se hizo por medios espectrofotométricos (Hassid y Neufeld 1964). Algunas plantas que fueron dañadas a lo largo del experimento se excluyeron de los análisis. Los datos fueron analizados por medio de ANOVA de dos vías (Crawley 2007), se verificó que los residuales cumplieran con los supuestos del análisis. Los análisis se llevaron a cabo con el paquete estadístico R ( R Development Core Team, 2009).

\section{Resultados}

Typha domingensis respondió a la adición de nitrógeno para todas las variables de respuesta y para ninguna respondió 
Cuadro 1. Respuesta de Typha domingensis y Schoenoplectus americanus a los tratamientos de fertilización. La concentración corresponde a litros de medio de cultivo, los datos son medias y desviaciones estándar.

\begin{tabular}{|c|c|c|c|c|c|c|c|c|}
\hline \multicolumn{9}{|c|}{ Typha domingensis } \\
\hline $\begin{array}{l}\mathrm{KNO}_{3} \\
(\mathrm{~g} / \mathrm{l})\end{array}$ & $\begin{array}{l}\mathrm{KH}_{2} \mathrm{PO}_{4} \\
(\mathrm{~g} / \mathrm{l})\end{array}$ & $\begin{array}{l}\text { Altura del tallo } \\
\text { más alto }(\mathrm{cm})\end{array}$ & $\begin{array}{c}\text { Altura } \\
\text { promedio }(\mathrm{cm})\end{array}$ & $\begin{array}{l}\text { Número de } \\
\text { tallos }\end{array}$ & $\begin{array}{l}\text { Biomasa } \\
\text { aérea (g) }\end{array}$ & $\begin{array}{l}\text { Biomasa de } \\
\text { rizomas (g) }\end{array}$ & $\begin{array}{l}\text { \% de } \\
\text { almidón }\end{array}$ & $\begin{array}{l}\text { Relación } \\
\text { raíz-tallo }\end{array}$ \\
\hline 0 & 0 & $125.4 \pm 5.8$ & $102.0 \pm 4.6$ & $1.3 \pm 0.1$ & $17.7 \pm 4.8$ & $14.8 \pm 3.1$ & $19.1 \pm 3.1$ & $1.8 \pm 2.6$ \\
\hline 0 & 0.015 & $117.2 \pm 7.2$ & $94.8 \pm 8.2$ & $2.0 \pm 0.2$ & $20.0 \pm 3.4$ & $20.2 \pm 7.1$ & $49.5 \pm 0.8$ & $0.8 \pm 0.4$ \\
\hline 0 & 0.03 & $117.8 \pm 6.9$ & $101.2 \pm 6.2$ & $1.6 \pm 0.2$ & $21.0 \pm 3.1$ & $17.4 \pm 1.8$ & $17.6 \pm 1.9$ & $0.9 \pm 0.3$ \\
\hline 0.03 & 0 & $151.3 \pm 5.1$ & $113.4 \pm 4.6$ & $2.3 \pm 0.2$ & $31.1 \pm 4.1$ & $29.2 \pm 1.2$ & $19.0 \pm 2.2$ & $1.2 \pm 0.9$ \\
\hline 0.03 & 0.015 & $153.5 \pm 5.2$ & $116.2 \pm 5.1$ & $2.2 \pm 0.4$ & $39.7 \pm 3.7$ & $26.2 \pm 0.4$ & $8.0 \pm 1.2$ & $0.7 \pm 0.4$ \\
\hline 0.03 & 0.03 & $138.3 \pm 7.0$ & $108.4 \pm 5.4$ & $2.1 \pm 0.2$ & $32.0 \pm 2.7$ & $22.7 \pm 1.8$ & $31.1 \pm 1.8$ & $0.7 \pm 0.3$ \\
\hline 0.06 & 0 & $168.0 \pm 5.0$ & $117.8 \pm 5.9$ & $2.0 \pm 0.3$ & $46.8 \pm 6.4$ & $35.4 \pm 0.8$ & $20.2 \pm 0.4$ & $0.9 \pm 0.4$ \\
\hline 0.06 & 0.015 & $163.3 \pm 5.9$ & $124.2 \pm 4.8$ & $2.7 \pm 0.2$ & $46.3 \pm 6.4$ & $40.9 \pm 2.2$ & $21.8 \pm 1.8$ & $0.9 \pm 0.4$ \\
\hline 0.06 & 0.03 & $168.4 \pm 6.2$ & $121.0 \pm 6.7$ & $3.0 \pm 0.2$ & $46.4 \pm 4.8$ & $40.7 \pm 1.9$ & $28.5 \pm 7.1$ & $1.0 \pm 0.9$ \\
\hline & \multicolumn{8}{|c|}{ Schoenoplectus americanus } \\
\hline $\begin{array}{l}\mathrm{KNO}_{3} \\
(\mathrm{~g} / \mathrm{l})\end{array}$ & $\begin{array}{l}\mathrm{KH}_{2} \mathrm{PO}_{4} \\
(\mathrm{~g} / \mathrm{l})\end{array}$ & $\begin{array}{l}\text { Altura del tallo } \\
\text { más alto }(\mathrm{cm})\end{array}$ & $\begin{array}{c}\text { Altura } \\
\text { promedio }(\mathrm{cm})\end{array}$ & $\begin{array}{l}\text { Número de } \\
\text { tallos }\end{array}$ & $\begin{array}{l}\text { Biomasa } \\
\text { aérea (g) }\end{array}$ & $\begin{array}{l}\text { Biomasa de } \\
\text { rizomas (g) }\end{array}$ & $\begin{array}{l}\text { \% de } \\
\text { almidón }\end{array}$ & $\begin{array}{l}\text { Relación } \\
\text { raíz-tallo }\end{array}$ \\
\hline 0 & 0 & $78.6 \pm 2.1$ & $68.7 \pm 1.8$ & $11.4 \pm 0.7$ & $4.9 \pm 0.3$ & $9.2 \pm 2.1$ & $11.2 \pm 3.3$ & $1.9 \pm 1.3$ \\
\hline 0 & 0.015 & $76.8 \pm 4.2$ & $67.0 \pm 3.9$ & $16.0 \pm 2.5$ & $6.2 \pm 1.1$ & $5.4 \pm 0.8$ & $23.9 \pm 7.8$ & $1.1 \pm 0.3$ \\
\hline 0 & 0.03 & $76.3 \pm 2.1$ & $72.5 \pm 1.7$ & $16.8 \pm 3.9$ & $6.5 \pm 0.5$ & $7.5 \pm 1.0$ & $19.2 \pm 5.3$ & $1.1 \pm 0.3$ \\
\hline 0.03 & 0 & $98.2 \pm 3.6$ & $79.6 \pm 2.2$ & $21.3 \pm 2.1$ & $11.7 \pm 1.1$ & $15.1 \pm 2.7$ & $18.9 \pm 4.3$ & $1.6 \pm 1.4$ \\
\hline 0.03 & 0.015 & $99.2 \pm 2.8$ & $79.4 \pm 1.9$ & $24.8 \pm 1.6$ & $17.0 \pm 1.3$ & $12.4 \pm 2.0$ & $15.7 \pm 3.8$ & $0.8 \pm 0.2$ \\
\hline 0.03 & 0.03 & $97.1 \pm 2.6$ & $76.5 \pm 1.7$ & $24.8 \pm 1.8$ & $17.0 \pm 1.2$ & $16.4 \pm 2.3$ & $20.5 \pm 2.5$ & $1.0 \pm 0.2$ \\
\hline 0.06 & 0 & $111.9 \pm 2.3$ & $86.2 \pm 1.7$ & $30.3 \pm 1.9$ & $23.3 \pm 1.6$ & $19.7 \pm 3.9$ & $16.2 \pm 2.4$ & $0.9 \pm 0.5$ \\
\hline 0.06 & 0.015 & $114.8 \pm 2.5 \mathrm{C}$ & $85.9 \pm 2.1 \mathrm{C}$ & $29.0 \pm 1.1 \mathrm{~B}$ & $27.7 \pm 1.3$ & $16.8 \pm 2.6$ & $12.7 \pm 2.8$ & $0.6 \pm 0.4$ \\
\hline 0.06 & 0.03 & $111.8 \pm 2.8 \mathrm{C}$ & $85.6 \pm 2.0 \mathrm{C}$ & $29.0 \pm 2.5 \mathrm{~B}$ & $24.4 \pm 2.6$ & $16.6 \pm 3.2$ & $11.9 \pm 1.8$ & $0.8 \pm 0.7$ \\
\hline
\end{tabular}

a la adición de fósforo (Cuadro 1). En particular la altura, evaluada tanto como altura del tallo más alto $\left(F_{(2,89)}=38, P\right.$ $<0.0001)$ o como altura promedio $\left(F_{(2,89)}=8, P=0.0006\right)$, aumentó en la medida en que aumentaba la concentración de nitrógeno (altura del tallo más alto: $123 \pm 22,146 \pm 20$ y $167 \pm 19 \mathrm{~cm}$; altura promedio: $102 \pm 21,111 \pm 16$ y $121 \pm$ $19 \mathrm{~cm})$. También el número de tallos $(1.6 \pm 0.7,2.1 \pm 1.0 \mathrm{y}$ $\left.2.5 \pm 0.91 ; F_{(2,89)}=9.9, P=0.0001\right)$ y la biomasa aérea $(20.1$ $\pm 13.3,33.8 \pm 12.5$ y $\left.47.3 \pm 20.3 ; F_{(2.89)}=22.8, P<0.0001\right)$, respondieron de la misma manera a la adición de nitrógeno. La acumulación de biomasa en los rizomas respondió de manera muy marcada a la adición de nitrógeno $(17.4 \pm 11.5$, $26.1 \pm 13.4$ y $\left.40.4 \mathrm{~g} \pm 19.6 ; F_{(2.89)}=17.8, P<0.000001\right)$, debido a que el tratamiento con mayor concentración de nitrógeno más que duplicó la biomasa en los rizomas comparado con los otros tratamientos. El almidón en los rizomas expresado en porcentaje con respecto al peso seco del rizoma respondió a la adición de ambos nutrimentos $\left(\mathrm{N}: F_{(2.89)}=\right.$ 7.3, $P=0.001 ;$ P: $\left.F_{(2,89)}=5.4, P<0.006\right)$ y la interacción fue también significativa $\left(F_{(2,89)}=26.2, P<0.000001\right)$, debido a que el tratamiento con la concentración baja de fósforo presento el mayor porcentaje de almidón $(50.3 \pm 24.8 \%)$ seguido por el tratamiento con la dosis alta y combinada de nitrógeno y fósforo $(32.8 \pm 1.5 \%)$. Para $T$. domingensis la relación entre la biomasa de raíces y de tallos no varió signi- ficativamente con los tratamientos de fertilización.

Schoenoplectus americanus respondió solamente a la adición de nitrógeno para las variables altura máxima (76.5 $\pm 11.4,99.9 \pm 10.0$ y $113.3 \mathrm{~cm} \pm 7.6 ; F_{(2.69)}=85.9, P<$ $0.000001)$, altura promedio $(68.1 \pm 10.3,80.5 \pm 6.5$ y $86.1 \pm$ $\left.6.0 \mathrm{~cm} ; F_{(2,69)}=35.6, P<0.000001\right)$ número de tallos $(13.7$ $\pm 6.0,23.5 \pm 6.9$ y $\left.29.2 \pm 7.0 ; F_{(2,69)}=32.6, P<0.0000001\right)$ y biomasa de rizomas $(7.6 \pm 4.8,14.9 \pm 6.8$ y $17.5 \pm 9.5$ $\mathrm{g} ; F_{(2,69)}=12.2, P<0.000001$ ). La biomasa aérea (Cuadro 1) respondió a la adición de nitrógeno $\left(F_{(2,69)}=85.9, P<\right.$ 0.000001 ) y también de fósforo (Cuadro $1 ; F_{(2,69)}=3.7, P=$ $0.03)$, y la interacción no fue significativa. La relación entre la biomasa de raíz y de tallos respondió también a ambos nutrimentos las diferencias no son tan marcadas lo que se refleja en los valores de significancia (nitrógeno; $F_{(2,69)}=$ $3.6, P=0.03$; fósforo; $\left.F_{(2,69)}=4.5, P=0.01\right)$, y la interacción no fue significativa.

\section{Discusión}

Las dos especies estudiadas respondieron principalmente a la adición de nitrógeno. Typha domingensis respondió a la adición de nitrógeno aumentando su biomasa aérea y la biomasa de los rizomas. De forma contrastante, fue el tratamiento de la dosis baja de fósforo el que acumuló más al- 
midón en los rizomas. Schoenoplectus americanus también respondió a la adición de nitrógeno aunque para el caso de la biomasa aérea la adición de fósforo también tuvo un efecto importante. Un aspecto importante es que para S. americanus, a diferencia de $T$. domingensis, ningún tratamiento de fertilización tuvo un efecto diferencial en la acumulación de almidón.

Estos resultados concuerdan en términos generales con los resultados del experimento de Escutia-Lara et al. (2009a) donde ambas especies se encontraban creciendo juntas. En ese caso, el crecimiento evaluado como altura y biomasa aérea de Typha domingensis respondió a la adición de nitrógeno pero no a la adición de fósforo y el crecimiento, en biomasa aérea, de $S$. americanus respondió a la adición de nitrógeno y fósforo. Con respecto a $T$. domingensis, estos resultados contrastan con aquellos que han mostrado una respuesta a la adición de fósforo en diferentes condiciones (Macek y Rejmmankova, 2007; Lorenzen et al., 2001; Miao et al., 2000). Los resultados confirman que Schoenoplectus americanus tiene la capacidad de almacenar almidón en los rizomas y que este representa entre el $11 \mathrm{y}$ el $24 \%$ del peso seco, siendo la segunda especie del género en la que se cuantifica almidón pues en $S$. lacustris el almidón puede representar hasta el 15\% del peso del rizoma (Steinmann y Brändle 1984).

En general, las plantas pueden responder en términos de su crecimiento a los niveles de nutrientes disponibles en el ambiente donde se desarrollan y esto es especialmente cierto en ambientes oligotróficos como muchos humedales alimentados por manantiales, como es el caso de nuestro sistema de referencia. La asimilación de nitratos por las raíces está mediada por la enzima nitrato reductasa. Aunque existen niveles constitutivos de esta enzima, un incremento de su actividad se puede inducir por diversos factores como luz y un aumento en la disponibilidad de nitrógeno (Taiz y Zeiger 2006). En este último caso, debido a que la asimilación de nitrógeno es un proceso energéticamente costoso, la inducción de la enzima sólo en los casos en los que el nutriente está disponible parece ser una adaptación que ha permitido a las especies vegetales reducir su actividad metabólica hasta el momento en el que los nutrientes estén disponibles (Taiz y Zeiger 2006). Para el caso de las hidrófitas consideradas en este estudio, es posible que la inducción de la nitrato reductasa ocurra de manera más expedita en Typha domingensis comparada con Schoenoplectus americanus, aspecto que debe ser estudiado en futuras investigaciones. Por otro lado, la asimilación de nitratos por medio de la nitrato reductasa se puede saturar, en función de la especie, en forma relativamente rápida. Sin embargo, al aumentar los niveles de nitrógeno en el suelo, por ejemplo, como resultado de lixiviados de actividades agrícolas, la disponibilidad de fósforo disminuye, con lo que el desarrollo de las plantas podría verse limitado, lo cual se compensó mediante fertilización en algunos tratamientos de este estudio (Arróniz-
Crespo et al. 2008). El hecho de que T. domingensis no haya respondido a la adición de fósforo pero sí a incrementos en la disponibilidad de nitrógeno, en contraste con S. americanus, que acumuló más peso seco con la fertilización con fósforo, sugiere que esa especie es más eficiente en la asimilación de este nutriente aún en condiciones limitantes, con lo cual se podría explicar, en parte, el éxito que ha tenido como especie invasora (Woo y Zedler 2002).

Las concentraciones de los nutrimentos en los humedales, en particular los macronutrimentos nitrógeno y fósforo, varían en función de la dinámica del suelo de la cuenca en la que se encuentran los humedales y de la posición de éstos dentro de la misma cuenca (Cronk y Fennessy, 2001). Por lo tanto, las alteraciones humanas en las cuencas, particularmente el cambio de uso de suelo y la agricultura, pueden alterar las concentraciones de estos macronutrimentos en los humedales y en consecuencia su dinámica (Zedler, 2003). Los resultados de este estudio sugieren que si se incrementan las concentraciones de nitrógeno en humedales en donde coexisten Typha domingensis y Schoenoplectus americanus la primera podría desplazar a la segunda, causando pérdida de la biodiversidad de los mismos (Escutia-Lara et al., 2009b).

\section{Agradecimientos}

Este proyecto fue financiado por la Universidad Nacional Autónoma de México por medio del proyecto PAPIIT IN203608 y CONACYT (SEP-CONACyT 2008-101335). Agradecemos a dos revisores anónimos sus comentarios y sugerencias que mejoraron considerablemente la versión final del manuscrito.

\section{Litertura citada}

Arróniz-Crespo M., Leake J.R., Horton P. y Phoenix G.K. 2008. Bryophyte physiological responses to, and recovery from, longterm nitrogen deposition and phosphorus fertilisation in acidic grassland. New Phytologist 180:864-874.

Craft C.B., Vymazal J. y Richardson C.J. 1995. Response of Everglades plant-communities to nitrogen and phosphorus additions. Wetlands 15:258-271.

Crawley R., 2007. The R book. John Wiley and Sons, Ltd., USA.

Cronk J.K. y Fennessy M.S. 2001. Wetland plants, biology and ecology. Lewis Publishers. Boca Raton, Florida.

Escutia-Lara Y., Gómez-Romero M. y Lindig-Cisneros R. 2009a. Nitrogen and phosphorus effect on Typha domingensis Presl. rhizome growth in a matrix of Schoenoplectus americanus (Pers.) Volkart ex Schinz and Keller. Aquatic Botany 90:74-77.

Escutia-Lara, Y., Lara-Cabrera S., Lindig-Cisneros, R.A. 2009b. Fire and dynamics of the emergent hydrophytes of the Mintzita wetlands, Michoacan, Mexico. Revista Mexicana de Biodiversidad 3:771-778.

Flores-Verdugo F., Moreno-Casasola P., Agraz-Hernández M.C., López-Rosas H., Benítez-Pardo D. y Travieso-Bello A.C. 2007. La topografía y el hidroperíodo: dos factores que condicionan 
la restauración de humedales costeros. Boletín de la Sociedad Botánica de México 80s:33-47.

Galatowitsch S.M., Anderson N.O. y Ascher P.D.. 1999. Invasiveness in wetland plants in temperate North America. Wetlands 19:733-755.

Hassid W.Z. y Neufeld E.F. 1964. Quantitative determination of starch in plant tissues. En: Whistler R.L. Ed. Methods in carbohydrate chemistry Vol. 4, pp. 33-36, Academic Press, New York.

Lara-Lara J.R., Arreola-Lizárraga J.A., Calderón-Aguilera L.E., Camacho-Ibar V.F., de la Lanza-Espino G., Escofet-Giansone A., Espejel-Carbajal M.I., Guzmán-Arroyo M., Ladah L.B., López-Hernández M., Meling-López E.A., Moreno-CasasolaBarceló P., Reyes-Bonilla H., Ríos-Jara E. y Zertuche-González J.A. 2008. Los ecosistemas costeros, insulares y epicontinentales. En Soberón J. Halffter G. y Llorente-Bousquets J. Comp. Capital natural de México, vol. I : Conocimiento actual de la biodiversidad. pp. 109-134. Conabio, México.

Lorenzen B., Brix H., Mendelssohn I.A., McKee K.L. y Miao S.L. 2001. Growth, biomass allocation and nutrient use efficiency in Cladium jamaicense and Typha domingensis as affected by phosphorus and oxygen availability. Aquatic Botany 70:117133.

Macek P. y Rejmánková E. 2007. Response of emergent macrophytes to experimental nutrient and salinity additions. Functional Ecology 21:478-488.

Miao S.L., Newman S. y Sklar F.H. 2000. Effects of habitat nutrients and seed sources on growth and expansion of Typha domingensis. Aquatic Botany 68:297-311.

Montes-Cartes C.G., Castillo-Agüero S. y López-Portillo J. 1999. Distribución del manglar en cuatro sistemas lagunares de la costa de Chiapas, México. Boletín de la Sociedad Botánicade México 64:25-34.

Nuñez Escobar, R. 2007. El suelo como medio natural en la nutrición de cultivos. En: González-Alcántar G. y Trejo-Téllez L.I.
Eds. Nutrición de Cultivos. pp. 93-157 Colegio de Posgraduados-Mundiprensa. México D.F.

Peralta-Pelaez, L.A. y Moreno-Casasola P. 2009. Floristic composition and diversity of wetland vegetation in dune lakes in Veracruz. Boletín de la Sociedad Botánica de México 85:89-101.

R Development Core Team, 2009. R: A Language and Environment for Statistical Computing. R Foundation for Statistical Computing, Vienna, Austria. <www.R-project.org>

Ramos-Ventura L.J. y Novelo-Retana A. 1993. Vegetación y flora acuáticas de la laguna de Yuriria, Guanajuato, México. Acta Botánica Mexicana 25:61-79.

Richardson J.L. y Vepraskas M.J. 2001. Wetland Soils. Lewis Publishers. Boca Raton, Florida.

Rojas-Moreno J. y Novelo-Retana A. 1995. Flora y vegetación acuáticas del lago de Cuitzeo, Michoacán, México. Acta Botánica Mexicana 31:1-17.

Shih J.G. y Finkelstein S.A. 2008. Range dynamics and invasive tendencies in Typha latifolia and Typha angustifolia in Eastern North America derived from herbarium and pollen records. Wetlands 28:1-16.

Steinmann F. y Brändle R. 1984. Carbohydrate and protein metabolism the rhizomes of the bulrush (Schoenoplectus lacustris (L.) Palla) in relation to natural development of the whole plant. Aquatic Botany 19:53-63.

Taiz L. y Zeiger E. 2006. Plant Physiology, Sinauer Press, Sunderland, MA.

Woo I. y Zedler J.B. 2002. Can nutrients alone shift a sedge meadow towards dominance by the invasive Typha $\times$ glauca? Wetlands 22:509-521.

Zedler J.B. 2003. Wetlands at your service: reducing impacts of agriculture at the watershed scale. Frontiers in Ecology and the Environment 1:65-72.

Zedler J.B. y Kercher S. 2004. Causes and Consequences of Invasive Plants in Wetlands: Opportunities, Opportunists, and Outcomes. Critical Reviews in Pant Sciences 23:431-452.

Recibido: 5 de febrero de 2010

Aceptado: 15 de agosto de 2010 